\title{
Mechanism of p38 MAP kinase activation in vivo
}

\author{
Deborah Brancho, ${ }^{1,5}$ Nobuyuki Tanaka, ${ }^{2,5,6}$ Anja Jaeschke, ${ }^{1,5}$ Juan-Jose Ventura, ${ }^{1,5}$ Nyaya Kelkar, ${ }^{1,5}$ \\ Yoshinori Tanaka, ${ }^{3}$ Masanao Kyuuma, ${ }^{4}$ Toshikazu Takeshita, ${ }^{4}$ Richard A. Flavell, ${ }^{2}$ \\ and Roger J. Davis ${ }^{1,7}$ \\ ${ }^{1}$ Howard Hughes Medical Institute and Program in Molecular Medicine, University of Massachusetts Medical School, \\ Worcester, Massachusetts 01605, USA; ${ }^{2}$ Howard Hughes Medical Institute and Section of Immunobiology, Yale University \\ Medical School, New Haven, Connecticut 06520, USA; ${ }^{3}$ Department of Microbiology, Tohoku University School of \\ Medicine, Sendai 980-8575, Japan; ${ }^{4}$ Department of Microbiology, Shinshu University School of Medicine, Matsumoto \\ 390-8621, Japan
}

The p38 mitogen-activated protein kinase (MAPK) is activated in vitro by three different protein kinases: MKK3, MKK4, and MKK6. To examine the relative roles of these protein kinases in the mechanism of $\mathrm{p} 38$ MAP kinase activation in vivo, we examined the effect of disruption of the murine Mkk3, Mkk4, and Mkk6 genes on the p38 MAPK signaling pathway. We show that MKK3 and MKK6 are essential for tumor necrosis factor-stimulated p38 MAPK activation. In contrast, ultraviolet radiation-stimulated p38 MAPK activation was mediated by MKK3, MKK4, and MKK6. Loss of p38 MAPK activation in the mutant cells was associated with defects in growth arrest and increased tumorigenesis. These data indicate that p38 MAPK is regulated by the coordinated and selective actions of three different protein kinases in response to cytokines and exposure to environmental stress.

[Keywords: MAP kinase; p38; JNK; MKK3; MKK4; MKK6]

Received April 28, 2003; revised version accepted June 17, 2003.

Several groups of mitogen-activated protein kinase (MAPK) signal transduction pathways have been identified in mammals, including extracellular signal-regulated protein kinase (ERK), c-Jun $\mathrm{NH}_{2}$-terminal kinase (JNK), and p38 MAPK. Each of these groups of MAPK is activated by dual phosphorylation on Thr and Tyr within a tripeptide motif (Thr-Xaa-Tyr) located within the activation loop of the MAPK. This phosphorylation is mediated by seven MAPK kinases (MAPKKs) that have specificity for individual MAPK isoforms. Thus, ERK1 and ERK2 are activated by MEK1 and MEK2, ERK5 is activated by MEK5, JNK is activated by MKK4 and MKK7, and p38 MAPK is activated by MKK3 and MKK6 (Schaeffer and Weber 1999; Kyriakis and Avruch 2001). These MAPKKs and MAPKs can create independent signaling modules that may function in parallel.

The mechanism that accounts for the specificity of MAPKKs to activate individual MAPK isoforms is mediated, in part, by an interaction between an $\mathrm{N}$-terminal region located on the MAPKK and a docking site located

\footnotetext{
${ }^{5}$ These authors contributed equally to this work.

${ }^{6}$ Present address: Department of Microbiology and Immunology, Tohoku, University School of Medicine, Sendai 980-8575, Japan.

${ }^{7}$ Corresponding author.

E-MAIL Roger.Davis@umassmed.edu; FAX (508) 856-3210.

Article published online ahead of print. Article and publication date are at http://www.genesdev.org/cgi/doi/10.1101/gad.1107303.
}

on the MAPK (Bardwell and Thorner 1996; Enslen and Davis 2001). Recently, structural insight into the mechanism of interaction between a MAPKK and a MAPK has been achieved by X-ray crystallography (Chang et al. 2002). This analysis demonstrated that there is a direct interaction of the $\mathrm{N}$-terminal region of the MAPKK with a docking groove present on the surface of the MAPK distant from the catalytic active site (Weston et al. 2002). A second determinant of MAPKK specificity is the structure of the MAPK activation loop that contains the ThrXaa-Tyr dual phosphorylation motif (Enslen et al. 2000). The specificity of these interactions mediate, in part, the ability of an individual MAPKK to activate a particular MAPK selectively.

It is interesting that mammalian MAPK signaling modules include more than one MAPKK because in yeast only a single MAPKK appears to activate each MAPK. The role of this pathway complexity in mammals is unclear. However, it may be significant that individual yeast MAPK isoforms are activated by only a limited group of extracellular stimuli, but mammalian MAPK isoforms are activated by a wide array of extracellular stimuli. It is therefore possible that the employment of more than one MAPKK for the activation of a specific mammalian MAPK may contribute to the ability of these signaling modules to respond to multiple stimuli. 
Studies of the ERK1/2 signaling module demonstrate that MEK1 has an essential function in placental formation during embryonic development, but the role of MEK1 to activate ERK1/2 appears to be largely redundant with MEK2 (Giroux et al. 1999). In contrast, studies of the JNK signaling module have demonstrated nonredundant functions of both MKK4 and MKK7 in JNK activation. JNK is phosphorylated preferentially on Tyr by MKK4, whereas MKK7 preferentially phosphorylates JNK on Thr (Lawler et al. 1998; Tournier et al. 2001; Wada et al. 2001). Because dual phosphorylation on Thr and Tyr is required for full activation of JNK (Derijard et al. 1994), these data suggest that MKK4 and MKK7 may cooperate to activate JNK. Strong support for this conclusion has been obtained from studies of $M k k 4$ and $M k k 7$ gene disruption. Ultraviolet (UV) radiation causes activation of both MKK4 and MKK7 (Tournier et al. 1999|, and loss-of-function mutations in either Mkk4 or Mkk7 cause reduced UV-stimulated JNK activation (Nishina et al. 1997; Yang et al. 1997; Ganiatsas et al. 1998; Tournier et al. 2001; Wada et al. 2001; Kishimoto et al. 2003). Significantly, compound mutations of both $M k k 4$ and $M k k 7$ eliminated the ability of UV radiation to activate JNK, indicating that these MAPKK isoforms are essential for JNK activation (Tournier et al. 2001).

The observation that MKK4 and MKK7 have nonredundant functions in the activation of JNK suggests that other MAPKK may also have specialized functions in mammalian MAPK signaling modules. The purpose of the study reported here was to examine the role of MAPKK isoforms in the activation of p38 MAPK. Previous studies have identified the isoforms MKK3 and MKK6 as specific activators of p38 MAPK (Derijard et al. 1995; Raingeaud et al. 1996). However, in vitro studies suggest that MKK4, an activator of JNK, may also contribute to p38 MAPK activation (Derijard et al. 1995; Doza et al. 1995; Lin et al. 1995). Furthermore, it has also been reported that some cell surface receptors, including tumor necrosis factor (TNF) receptors, may activate p38 MAPK by a MAPKK-independent mechanism (Ge et al. 2002, 2003). Our approach was to examine the effect of loss-of-function mutations in the murine $M k k 3, M k k 4$, and Mkk6 genes. We show that all three MAPKK isoforms can contribute to p38 MAPK activation and that the repertoire of MAPKK isoforms that cause p38 MAPK activation in vivo depends on the specific stimulus that is examined. Loss of p38 MAPK regulation in the mutant cells causes defects in growth arrest and increased tumorigenesis.

\section{Results}

Targeted disruption of Mkk3 and Mkk6

We have previously reported phenotypes of mice with targeted disruptions of the $M k k 3$ and $M k k 6$ genes (Lu et al. 1999; Wysk et al. 1999; Tanaka et al. 2002). The $\mathrm{Mkk3}^{-{ }^{-}}$and $\mathrm{Mkk6}^{-/-}$mice were viable with no obvious developmental abnormalities. Mice with compound mutations in $M k k 3$ and $M k k 6$ were created by breeding these mutant mice. Because $M k k 3$ and Mkk6 are both located on mouse Chromosome 11, we screened mice for the presence of a chromosome containing disruptions of both $M k k 3$ and $M k k 6$. Subsequent breeding resulted in the generation of mice with compound mutations in Mkk3 and Mkk6. Mice lacking expression of both MKK3 and MKK6 were not viable. The $\mathrm{Mkk3}^{-/-} \mathrm{Mkk6}^{-1-}$ embryos died during midgestation at embryonic day 11.011.5 (E11.0-E11.5). Major defects in the formation of the placenta and deficiencies in the development of the embryonic vasculature were observed (Fig. 1). The mutant mice appeared to be developmentally delayed and exhibited symptoms of severe anoxia. This phenotype of $\mathrm{Mkk3}^{-/-} \mathrm{Mkk6}^{-/-}$mice resembles that previously described for p38 $\mathrm{MAPK}^{-/-}$embryos (Adams et al. 2000; Allen et al. 2000; Tamura et al. 2000). The similar embryonic phenotype of these mice is consistent with the known role of MKK3 and MKK6 to activate p38 MAPK selectively (Raingeaud et al. 1996). Together, these data indicate that MKK3 and MKK6 serve redundant roles that are essential for survival.

To characterize the effects of MKK3 and MKK6 deficiency biochemically, we isolated fibroblasts from wildtype and mutant embryos. Immunoblot analysis demonstrated that $\mathrm{Mkk3}^{-/-}$mice did not express MKK3, that $\mathrm{Mkk6}^{-/-}$cells did not express MKK6, and that $\mathrm{Mkk3}^{-/-}$ $\mathrm{Mkk6}^{-/-}$cells did not express MKK3 or MKK6 (Fig. 2A). In contrast, these cells expressed similar amounts of JNK, p38 MAPK, MKK4, and MKK7 (Fig. 2A). These data indicated that defects in the expression of MKK3 and MKK6 did not cause marked changes in the expression of other components of stress-activated MAP kinase pathways. Phase contrast microscopy demonstrated that the wild-type, $\mathrm{Mkk3}^{-/-}, \mathrm{Mkk6}^{-/-}$, and $\mathrm{Mkk3}^{-/-} \mathrm{Mkk6}^{-/-}$cells displayed the typical flattened appearance of embryonic fibroblasts (Fig. 2B). The rate of proliferation of these cells in medium supplemented with $10 \%$ fetal calf serum was similar, although the $\mathrm{Mkk3}^{-/-} \mathrm{Mkk6}^{-/-}$fibroblasts were found to reach a slightly higher saturation density compared with wild-type fibroblasts (Fig. 2C).

\section{MKK3 and MKK6 are essential for TNF $\alpha$-stimulated p38 MAPK activation}

To examine the role of MKK3 and MKK6 in p38 MAPK activation in vivo, we investigated the effect of TNF $\alpha$ on wild-type and mutant fibroblasts by immunoblot analysis using antibodies that bind phosphorylated and activated MAPK and MAPKK. Control studies using wildtype cells demonstrated that $\mathrm{TNF} \alpha$ caused a marked increase in MAPK activation (JNK, ERK, and p38; Fig. 3A) and a more moderate activation of both MKK3 and MKK6 (Fig. 3A). Mkk6 gene disruption did not cause obvious changes in MAPK activation in cells treated with $\mathrm{TNF} \alpha$. In contrast, $M k k 3$ gene disruption caused reduced activation of p38 MAPK, but did not alter JNK or ERK activation. Interestingly, compound mutant cells lacking both MKK3 and MKK6 were severely defective in TNF $\alpha$-stimulated p38 MAPK activation (Fig. 3A). This defect in TNF $\alpha$-stimulated p38 MAPK activation in 
A

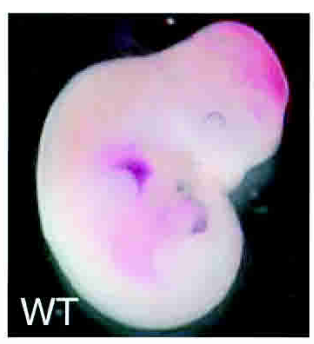

B

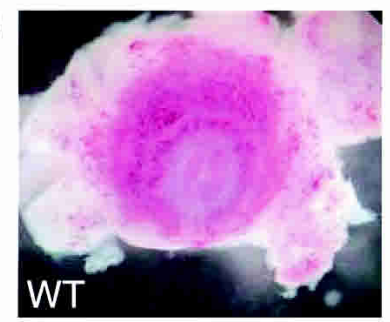

C

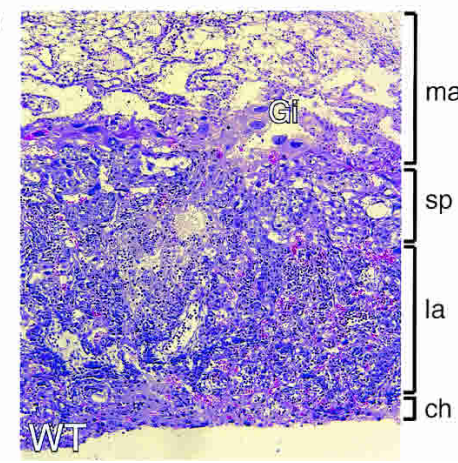

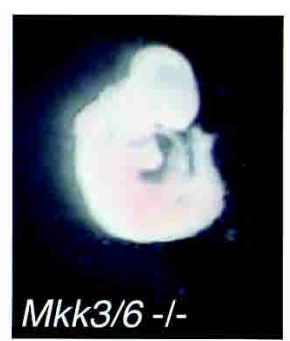
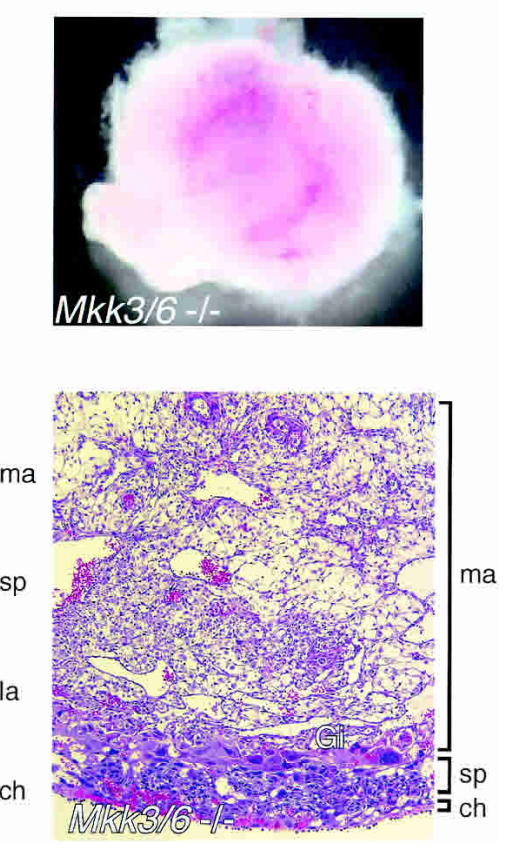

Figure 1. Characterization of $\mathrm{Mkk3}^{-/-} \mathrm{Mkk6}^{-/-}$mice. (A) The morphology of wild-type and $M k k 3 / 6^{-1-}$ embryos at E11.0 is illustrated. The mutant embryos are anemic and hypovascular. $(B)$ The morphology of the placenta of wild-type and $M k k 3 / 6^{-/-}$embryos at E11.0 is illustrated (ventral view). The placenta of the mutant embryos is pale with decreased vascularization. $(C)$ Sections of the placenta from wild-type and $M k k 3 / 6^{-/-} \mathrm{em}$ bryos at E10.5 were stained with hematoxylin and eosin (H\&E). The developing labyrinth and spongiotrophoblast layers are markedly decreased in the mutant compared with the wild type. (Ch) chorionic plate; (la) labyrinth; (sp) spongiotrophoblast; (ma) maternal decidual tissue; (Gi) trophoblast giant cells.
$\mathrm{Mkk3}^{-/-} \mathrm{Mkk6}^{-/-}$cells was confirmed by in vitro kinase assays (Fig. 3B). Together, these data indicate that MKK3 and MKK6 serve redundant, but essential, roles in the TNF $\alpha$-stimulated activation of p38 MAPK.

\section{MKK3 and MKK6 contribute to p38 MAPK activation caused by $U V$ radiation}

MKK3 and MKK6 are essential for TNF $\alpha$-stimulated p38 MAPK activation (Fig. 3). To investigate whether MKK3 and MKK6 are required for other stimuli that activate p38 MAPK, we examined the effect of an environmental stress, UV radiation. Control studies using immunoblot analysis with phospho-specific antibodies demonstrated that exposure of wild-type cells to UV radiation caused increased activation of MAPKK (MKK3, MKK4, and MKK6; Fig. 4A) and caused increased activation of ERK, JNK, and p38 MAPK (Fig. 4A). Deficiency of MKK3 or MKK6 did not cause marked defects in p38 MAPK activation. Strikingly, UV radiation also caused p38 MAP kinase activation in $M k k^{-/-} M k k 6^{-/-}$cells, although the extent of p38 MAPK activation was diminished compared with wild-type cells (Fig. 4A). This observation was confirmed using in vitro kinase assays (Fig. 4B). Together, these data indicate that although MKK3 and
MKK6 contribute to p38 MAPK activation in cells exposed to UV radiation, these protein kinases are not essential for UV-stimulated p38 MAPK activation (Fig. 4). This observation markedly contrasts with the essential role of MKK3 and MKK6 in TNF $\alpha$-stimulated p38 MAPK activation (Fig. 3).

\section{MKK4 contributes to UV-stimulated p38 MAPK activation}

The observation that $\mathrm{Mkk3}^{-/-} \mathrm{Mkk}^{-/-}$cells can respond to UV radiation with increased p38 MAPK activation demonstrated that a mechanism must exist in UVstimulated cells to activate p38 MAPK in the absence of MKK3 and MKK6. Previous studies have indicated two possible mechanisms. First, p38 MAPK may be activated by a MAPKK-independent mechanism. For example, it has been reported that the TAB1 adapter protein may cause p38 MAPK activation in the absence of MAPKK involvement (Ge et al. 2002, 2003). This TAB1-dependent (and MAPKK-independent) mechanism has been implicated in p38 MAPK activation caused by TNF $\alpha$ /Ge et al. 2002, 2003). However, because we now show that TNF $\alpha$-stimulated p38 MAPK requires MKK3 and MKK6 (Fig. 3), the conclusion that TNF $\alpha$ causes MAPKK-inde- 
A

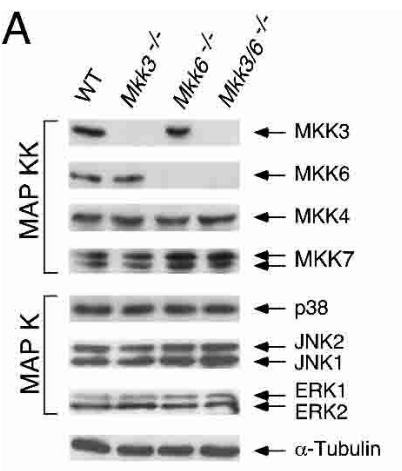

C

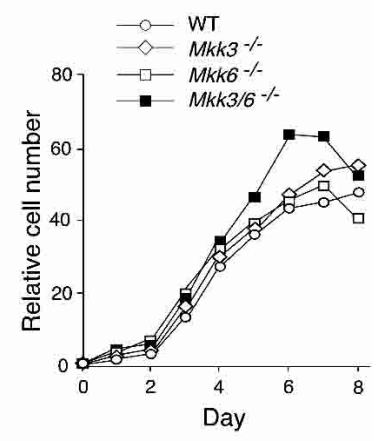

B

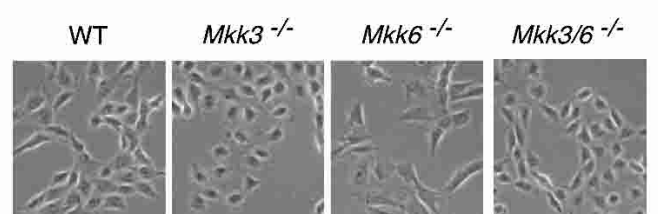

Figure 2. Isolation of fibroblasts from MKK3- and MKK6-deficient mice. (A) Wild-type (WT), $\mathrm{Mkk3}^{-/-}, \mathrm{Mkk6}^{-/-}$, and $\mathrm{Mkk3}^{-/-}$ $M k k 6^{-/-}$fibroblasts were cultured in vitro. Extracts prepared from these cells were examined by immunoblot analysis using antibodies to JNK, ERK, p38 MAPK, MKK3, MKK4, MKK6, MKK7, and $\alpha$-tubulin. (B) Cultures of wild-type (WT), $\mathrm{Mkk3}^{-/-}$, $\mathrm{Mkk6}^{-/-}$, and $\mathrm{Mkk3}^{-/-} \mathrm{Mkk6}^{-/-}$fibroblasts were examined by phase contrast microscopy. $(C)$ The proliferation of wild-type (WT), $\mathrm{Mkk3}^{-/-}, \mathrm{Mkk6}^{-/-}$, and $\mathrm{Mkk3}^{-/-} \mathrm{Mkk6}^{-/-}$fibroblasts cultured in medium supplemented with $10 \%$ fetal calf serum was examined. The relative cell number was measured by staining with crystal violet $\left(\mathrm{OD}_{595} \mathrm{~nm}\right)$. The normalized data presented are the mean of triplicate determinations and are representative of three independent experiments.

pendent activation of p38 MAP kinase (Ge et al. 2002, 2003) can be questioned. Given that TAB1 has not been implicated in p38 MAPK activation caused by UV radiation (Ge et al. 2002, 2003), it is unlikely that TAB1 contributes to the UV-stimulated activation of p38 MAPK observed in $\mathrm{Mkk3}^{-/-} \mathrm{Mkk6}^{-/-}$fibroblasts (Fig. 4). A second possible mechanism that may account for MKK3and MKK6-independent activation of p38 MAPK is that these protein kinases may not be the only MAPKKs that can activate p38 MAPK. For example, in vitro studies indicate that MKK4, an established activator of JNK, may also activate p38 MAPK (Derijard et al. 1995; Doza et al. 1995; Lin et al. 1995).

To test the role of MKK4 in the UV-stimulated activation of $\mathrm{p} 38 \mathrm{MAPK}$, we compared the activation of $\mathrm{p} 38$ MAPK in wild-type and $\mathrm{MkK}^{4^{-/}}$fibroblasts. As expected (Nishina et al. 1997; Yang et al. 1997; Ganiatsas et al. 1998; Tournier et al. 2001), Mkk4 gene disruption caused decreased activation of JNK following exposure to UV or TNF $\alpha$ (Fig. 5A). In contrast, the loss of MKK4 expression caused no marked decrease in p38 MAPK activation in response to UV or TNF $\alpha$ (Fig. 5B). These data confirm the conclusion that MKK4 has a nonredundant role in the activation of JNK and demonstrate that MKK4 has either no role, or a redundant role, in the activation of $\mathrm{p} 38$ MAPK.
To test whether MKK4 may have a redundant role in the activation of p38 MAPK, we examined the effect of MKK4 loss of function in wild-type and $\mathrm{Mkk}^{-/-}$ $M k k 6^{-/-}$fibroblasts. Attempts to construct triple knockout mice $\left(\mathrm{Mkk3}^{-/-} \mathrm{Mkk}^{-/-} \mathrm{Mkk6}^{-/-}\right)$by breeding were not successful, in part because all three genes are linked
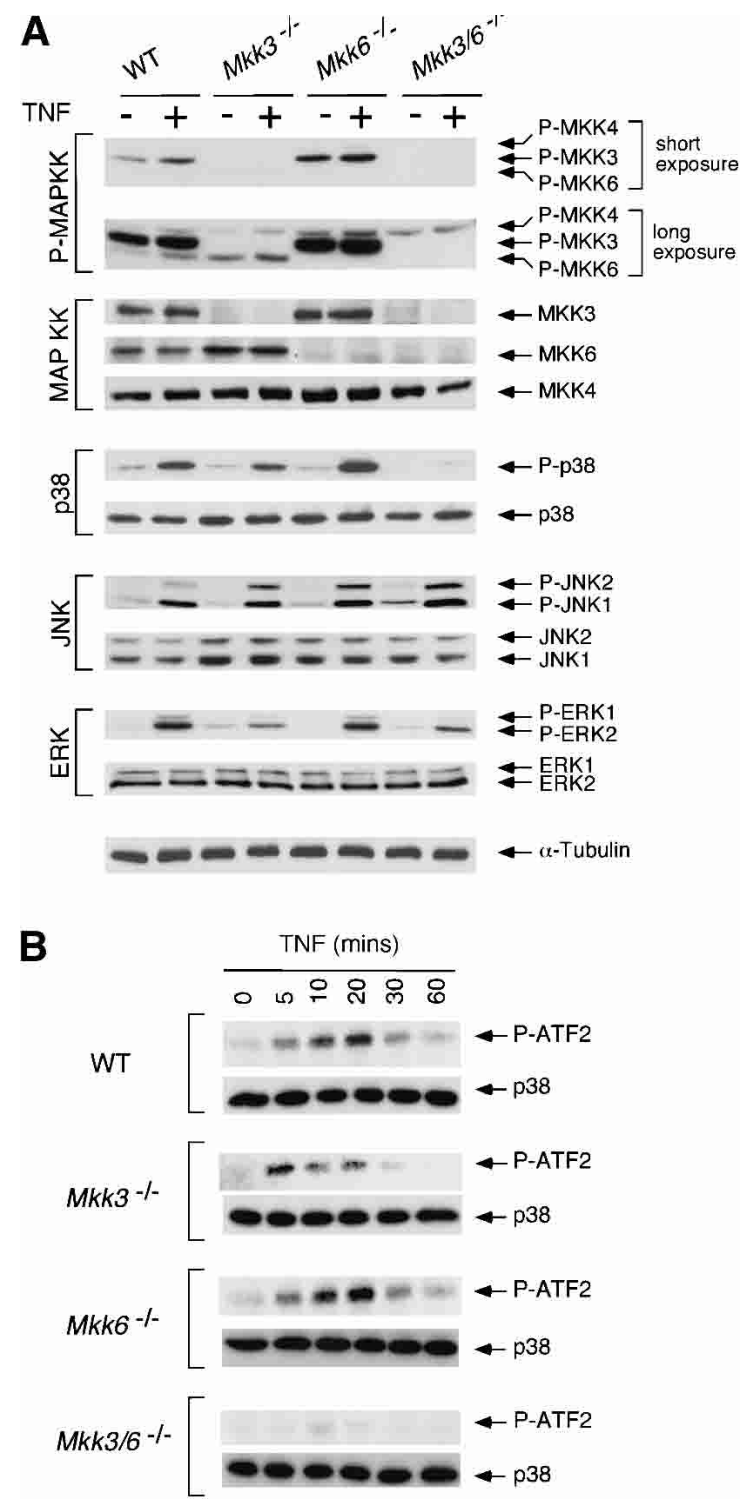

Figure 3. Targeted disruption of $M k k 3$ and $M k k 6$ prevents activation of p38 MAPK by tumor necrosis factor. (A) Wild-type (WT), $\mathrm{Mkk3}^{-1-}, \mathrm{Mkk6}^{-/-}$, and $\mathrm{Mkk3}^{-/-} \mathrm{Mkk6}^{-/-}$fibroblasts were treated without and with $10 \mathrm{ng} / \mathrm{mL} \mathrm{TNF} \alpha(10 \mathrm{~min})$. Extracts prepared from these cells were examined by immunoblot analysis using antibodies to phospho-MKK3/6 (P-MKK3, P-MKK4, P-MKK6), MKK3, MKK4, MKK6, phospho-p38 MAPK (P-p38), p38 MAPK, phospho-JNK (P-JNK), JNK, phospho-ERK (P-ERK), ERK, and $\alpha$-Tubulin. The phospho-MKK3/6 antibody binds activated MKK3 and MKK4 and can also bind more weakly to activated MKK6. Two exposures of the phospho-MKK3/6 immunoblot are presented to show activated MKK3, MKK4, and MKK6. (B) The activation of p38 MAPK was examined by in vitro kinase assays using ATF2 as the substrate. 
A
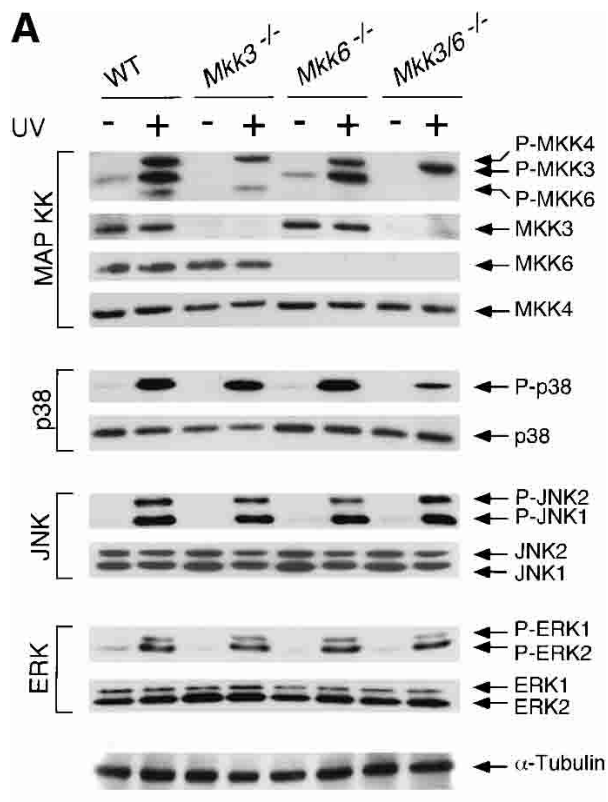

B
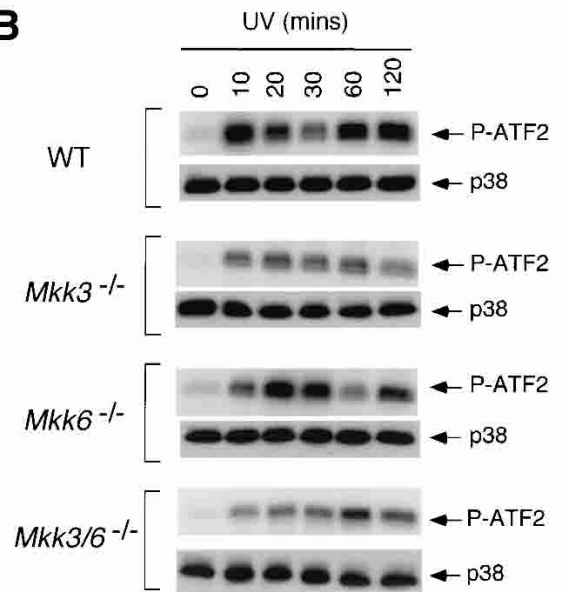

Figure 4. Targeted disruption of $M k k 3$ and $M k k 6$ does not prevent UV-stimulated activation of p38 MAPK. (A) Wild-type (WT), $\mathrm{Mkk3}^{-/-}, \mathrm{Mkk6}^{-/-}$, and $\mathrm{Mkk3}^{-1-} \mathrm{Mkk6}^{-1-}$ fibroblasts were treated without and with $60 \mathrm{~J} / \mathrm{m}^{2}$ of UV radiation and incubated (30 min). Extracts prepared from these cells were examined by immunoblot analysis using antibodies to phospho-MKK3/6 (PMKK3, P-MKK4, P-MKK6), MKK3, MKK4, MKK6, phospho-p38 MAPK (P-p38), p38 MAPK, phospho-JNK (P-JNK), JNK, phospho-ERK (P-ERK), ERK, and $\alpha$-Tubulin. ( $B$ ) The activation of p38 MAPK was examined by in vitro kinase assays using ATF2 as the substrate.

on mouse Chromosome 11. We therefore used an alternative approach to test the role of MKK4 in p38 MAPK activation using siRNA (Fig. 5C). Decreased expression of MKK4 caused by siRNA caused no change in UVstimulated activation of p38 MAPK in wild-type cells, but strongly suppressed p38 MAPK activation in $\mathrm{Mkk3}^{-/-} \mathrm{Mkk6}^{-/-}$fibroblasts (Fig. 5C). Similarly, inhibition of MKK4 with dominant-negative JNK caused no change in UV-stimulated p38 MAPK activation in wildtype cells, but inhibited p38 MAPK activation in
$M k k 3^{-/-} \mathrm{Mkk6}^{-/-}$fibroblasts (data not shown). Together, these data indicate that MKK4 serves a role that is redundant with MKK3 and MKK6 in the activation of p38 MAPK in cells exposed to UV radiation.

\section{Altered p38 MAPK regulation causes defects in growth} arrest and increased tumorigenesis

It is been proposed that the p38 MAPK pathway regulates growth arrest (Bulavin et al. 2002a). Because $\mathrm{Mkk3}^{-1-} \mathrm{Mkk6}^{-/-}$fibroblasts exhibit severe defects in p38 MAPK regulation, we investigated whether these cells might have altered proliferative responses. Control studies demonstrated that although p38 MAPK activation was markedly reduced in $\mathrm{Mkk}^{-/-} \mathrm{Mkk6}^{-/-}$fibroblasts (Fig. 2A), the rate of proliferation of wild-type and $\mathrm{Mkk3}^{-/-} \mathrm{Mkk6}^{-/-}$fibroblasts was similar (Fig. 2B). However, differences in proliferation between these cells were observed following serum starvation (Fig. 6B). Wildtype cells cultured in serum-free medium were not observed to proliferate. In contrast, the $\mathrm{Mkk3}^{-/-} \mathrm{Mkk6}^{-/-}$ fibroblasts were found to have increased proliferation potential in serum-free medium (Fig. 6B). To biochemically characterize the difference between the wild-type and $\mathrm{Mkk3}^{-1-} \mathrm{Mkk6}^{-/-}$fibroblasts, we examined $\mathrm{Rb}$ phosphorylation (a hallmark of G1/S progression) in serumstarved wild-type and mutant fibroblasts. Serum starvation caused $\mathrm{Rb}$ dephosphorylation in wild-type fibroblasts, but not in $\mathrm{Mkk}^{-/-} \mathrm{Mkk6}^{-/-}$fibroblasts (Fig. 6D). Cyclin-dependent protein kinases are thought to represent the major group of $\mathrm{Rb}$ kinases in vivo. We therefore examined cyclin expression in the wild-type and $\mathrm{Mkk3}^{-/-} \mathrm{Mkk6}^{-/-}$fibroblasts. Ribonuclease protection assays demonstrated that serum starvation caused decreased expression of D-type cyclins in wild-type cells (Fig. 6C). In contrast, the expression of D-type cyclins was maintained in serum-starved $\mathrm{Mkk3}^{-/-} \mathrm{MkkG}^{-1-}$ fibroblasts. Immunoblot analysis confirmed that D-type cyclins were selectively expressed in serum-starved $\mathrm{Mkk3}^{-1-} \mathrm{Mkk6}^{-/-}$fibroblasts, but not in the serumstarved wild-type fibroblasts (Fig. 6D). This deregulated expression of D-type cyclins in $\mathrm{Mkk3}^{-/-} \mathrm{Mkk6}^{-/-}$fibroblasts is consistent with the established role of p38 MAP kinase as an inhibitor of cyclin D gene expression (Lavoie et al. 1996).

The p38 MAP kinase pathway has also been implicated in the regulation of c-Jun gene expression (Han et al. 1997; Hazzalin et al. 1997). Defects in the p38 MAPK pathway in $\mathrm{Mkk3}^{-{ }^{--}} \mathrm{Mkk6}^{-/-}$fibroblasts could therefore cause disrupted c-Jun expression and consequently altered cellular proliferation. We therefore examined c-Jun mRNA expression in wild-type and $\mathrm{Mkk3}^{-/-} \mathrm{Mkk6}^{-/-} \mathrm{fi}-$ broblasts (Fig. 6E). Contrary to expectations, c-Jun expression was increased in $\mathrm{Mkk3}^{-{ }^{-}} \mathrm{Mkk6}^{-/-}$fibroblasts. These data indicate that although the MKK3/6 pathway may contribute to the regulation of c-Jun expression, this role of MKK3/6 can be compensated by the function of other signal transduction pathways in fibroblasts. Indeed, previous studies have demonstrated that c-Jun ex- 
Brancho et al.

A
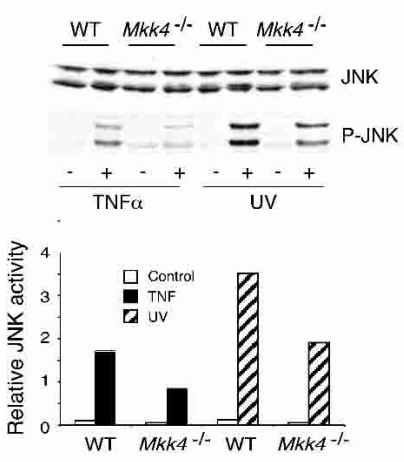

B
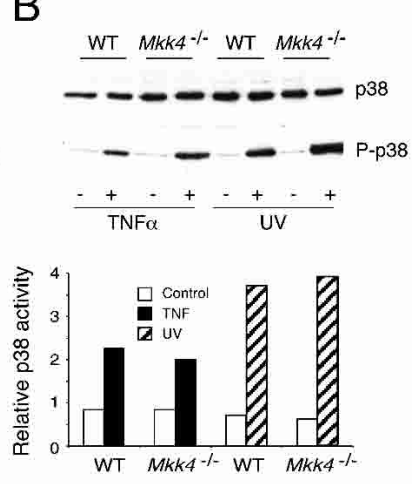

C
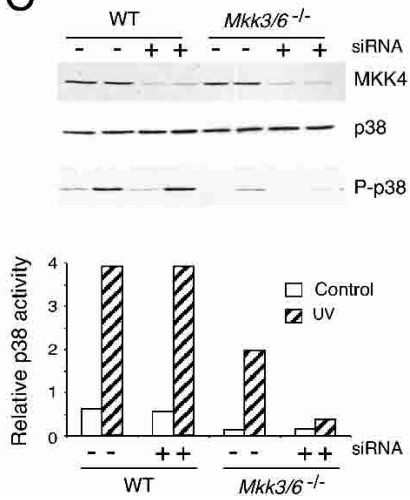

Figure 5. MKK4 deficiency causes decreased activation of JNK and p38 MAPK. $(A, B)$ Wild-type $\left(\right.$ WT) and $M k k 4^{-/-}$fibroblasts were treated without and with $10 \mathrm{ng} / \mathrm{mL}$ TNF $\alpha(15 \mathrm{~min})$ or $60 \mathrm{~J} / \mathrm{m}^{2}$ of UV radiation $(30 \mathrm{~min})$. The expression of JNK $(A)$ and $\mathrm{p} 38 \mathrm{MAPK}(B)$ was examined by immunoblot analysis (top panels). Activated JNK (P-JNK) and p38 (P-p38) were detected by immunoblot analysis (middle panels). JNK and p38 MAPK activity was also measured with an in vitro kinase assay using c-Jun and ATF2 as substrates, respectively (bottom panels). The amount of phosphorylated c-Jun and ATF2 was quantitated by PhosphorImager analysis (Molecular Dynamics). The data are presented in arbitrary units. (C) Wild-type (WT) and $M k k 3^{-1-} M k k 6^{-/}$fibroblasts were transfected with siRNA duplexes targeting MKK4 (+siRNA) or luciferase (-siRNA). The cells were treated without and with $60 \mathrm{~J} / \mathrm{m}^{2}$ of $\mathrm{UV} \mathrm{radiation}(30 \mathrm{~min})$ at $48 \mathrm{~h}$ posttransfection. The expression of MKK4, p38 MAPK, and activated p38 MAPK (P-p38) was examined by immunoblot analysis. p38 MAPK activity was examined in an in vitro kinase assay using the substrate ATF2. The amount of phosphorylated ATF2 was quantitated by PhosphorImager analysis.

pression is regulated by multiple functionally redundant signaling pathways (Chiariello et al. 2000).

Together, these data indicate that $\mathrm{Mkk3}^{-/-} \mathrm{Mkk6}^{-/-}$ fibroblasts exhibit a deregulated cell cycle associated with a failure to growth arrest in serum-free medium and altered expression of D-type cyclins and c-Jun (Fig. 6). Consistent with this conclusion, a dramatic increase in tumor burden was observed when SV40-large-T-antigenimmortalized $\mathrm{Mkk3}^{-/} \mathrm{Mkk6}^{-/}$fibroblasts (compared with wild-type cells) were injected subcutaneously in athymic nude mice (Fig. 7).

\section{Discussion}

The protein kinases MKK3 and MKK6 have been reported to specifically activate p38 MAP kinase (Derijard et al. 1995; Raingeaud et al. 1996). Consequently, these MAPKKs have been considered to be critical for p38 MAPK activation in vivo (Schaeffer and Weber 1999; Kyriakis and Avruch 2001). However, biochemical studies indicate that MKK4, an activator of JNK, can also activate p38 MAPK in vitro (Derijard et al. 1995; Doza et al. 1995; Lin et al. 1995). The physiological significance of this observation has been questioned. Indeed, the role of MKK4 in p38 MAPK activation in vivo is controversial because studies of $\mathrm{Mkk}^{-/-}$mice demonstrate major defects in JNK activation without obvious changes in p38 MAPK activation (Nishina et al. 1997; Yang et al. 1997). Furthermore, the proposed dual function of MKK4 to activate two separate groups of MAPK (JNK and p38) is unprecedented.

In this study, we have examined the role of MKK3, MKK4, and MKK6 using targeted gene disruption in mice and siRNA approaches. We show that all three
MAPKK isoforms contribute to the activation of p38 MAP kinase in cells exposed to UV radiation. These data confirm the importance of MKK3 and MKK6 in p38 MAP kinase activation. In addition, we demonstrate that MKK4 also contributes to p38 MAP kinase activation in cells exposed to UV radiation. This function of MKK4 was not detected in previous studies because the role of MKK4 in fibroblasts exposed to UV radiation is redundant with MKK3 and MKK6. Nevertheless, MKK4 is established by these data to be an activator of two different groups of MAPK: p38 and JNK.

\section{MKK3 and MKK6 are essential for TNF $\alpha$-stimulated p38 MAPK activation}

TNF $\alpha$ causes p38 MAP kinase activation in wild-type fibroblasts, but not in $\mathrm{Mkk3}^{-/-} \mathrm{Mkk6}^{-/-}$fibroblasts (Fig. 3). This observation demonstrates that TNF $\alpha$ causes $\mathrm{p} 38$ MAPK activation in a MAPKK-dependent manner. However, a significant question relates to the specificity of this response involving MKK3 and MKK6. Why is there no discernable contribution of MKK4 to TNF $\alpha$-stimulated p38 MAPK activation? One contributing factor may be the observation that TNF $\alpha$ activates MKK3, MKK6, and MKK7, but does not activate MKK4 (Fig. 3; Tournier et al. 1999, 2001). However, disruption of the Mkk4 gene does cause reduced TNF $\alpha$-stimulated JNK activity, indicating that basally active MKK4 is required for maximal TNF $\alpha$-stimulated JNK activation (Tournier et al. 2001). The effectiveness of MKK4 to activate JNK under these conditions may be accounted for by the observation that MKK7 primarily phosphorylates JNK on Thr 180, whereas MKK4 primarily phosphorylates JNK on Tyr 182. Interestingly, phosphoThr 180-JNK is the 
A

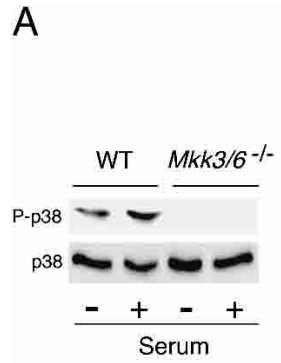

B

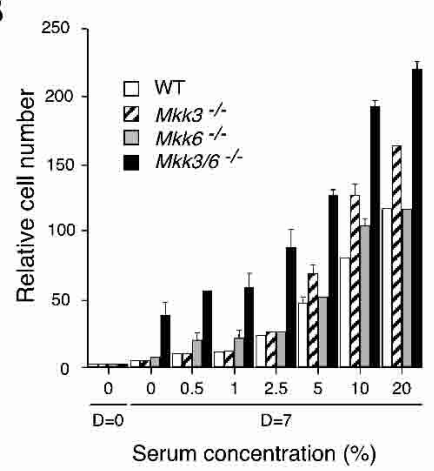

C

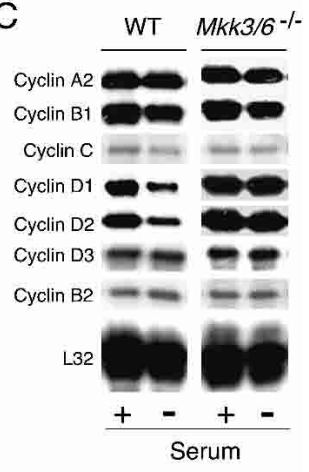

D

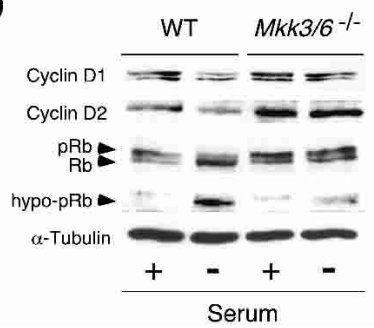

E

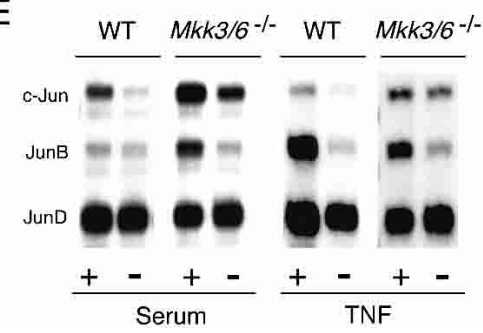

Figure 6. $\mathrm{Mkk3}^{-/-} \mathrm{Mkk6}^{-/-}$fibroblasts exhibit defects in growth arrest. (A) Wild-type (WT) and $\mathrm{Mkk3}^{-/-} \mathrm{Mkk6}^{-/-}$fibroblasts were serum starved $(24 \mathrm{~h})$. The effect of addition of $10 \%$ serum $(30 \mathrm{~min})$ to the serum-starved cells is presented. The expression of p38 MAPK and activated p38 MAPK (P-p38) was examined by immunoblot analysis. $(B)$ Wildtype (WT), $\mathrm{Mkk3}^{-/-}, \mathrm{Mkk6}^{-/-}$, and $\mathrm{Mkk3}^{-/-}$ $M k k 6^{-/-}$fibroblasts were cultured in different concentrations of fetal calf serum (7 d). The relative cell number was measured by staining with crystal violet $\left(\mathrm{OD}_{595} \mathrm{~nm}\right)$. The normalized data presented are the mean of triplicate determinations and are representative of three independent experiments. $(C)$ Cyclin and L32 mRNA expression in wild-type (WT) and $\mathrm{Mkk3}^{-/-} \mathrm{Mkk6}^{-/-}$fibroblasts was examined in a ribonuclease protection assay. Cells growing in $10 \%$ fetal calf serum were compared with cells cultured $(24 \mathrm{~h})$ in serum-free medium. $(D)$ Extracts prepared from wild-type (WT) and $\mathrm{Mkk3}^{-/-} \mathrm{Mkk6}^{-/-}$fibroblasts were examined by immunoblot analysis using antibodies to cyclin $\mathrm{D} 1$, cyclin $\mathrm{D} 2, \mathrm{Rb}$ (detects $\mathrm{Rb}$ and phospho- $\mathrm{Rb}$ ), hypo-pRb (detects hypo-phosphorylated $\mathrm{Rb}$ ), and $\alpha$-Tubulin. Cells growing in $10 \%$ fetal calf serum were compared with cells cultured $(24 \mathrm{~h})$ in serum-free medium. (E) c-Jun, JunB, and JunD mRNA expression in wild-type (WT) and $M k k 3^{-/-} M k k 6^{-/-}$ fibroblasts incubated without and with $10 \mathrm{ng} / \mathrm{mL}$ TNF $\alpha$ or $10 \%$ serum ( $24 \mathrm{~h}$ ) was examined in a ribonuclease protection assay.

preferred substrate for MKK4 compared with nonphosphorylated JNK (Lawler et al. 1998). The low Km of phosphoThr 180-JNK as a substrate for Tyr phosphorylation by MKK4 most likely accounts for the ability of basally active MKK4 to participate in TNF $\alpha$-stimulated JNK activation.

The mechanism of MKK4 activation of p38 MAPK is markedly different from that for the activation of JNK. MKK4 preferentially phosphorylates JNK on Tyr (Lawler et al. 1998), but phosphorylates p38 MAPK equally on Thr and Tyr (Doza et al. 1995; Tournier et al. 2001). Similarly, p38 MAPK is phosphorylated on both Thr and Tyr by MKK3 and MKK6 (Enslen et al. 2000). The absence of preferential Thr or Tyr phosphorylation of p38 MAPK may contribute to the lack of a role for MKK4 in TNF $\alpha$-stimulated p38 MAPK activation.

\section{Activation of p38 MAP kinase by} MAPKK-independent mechanisms

Our studies of fibroblasts have not revealed a role for a MAPKK-independent mechanism of p38 MAPK activation. However, it is possible that such mechanisms of p38 MAPK activation may exist in other cell types. Similarly, MAPKK-independent mechanisms of p38 MAPK activation may be present in fibroblasts exposed to specific stimuli. Recent studies have established that the adapter protein TAB1 represents an example of a mechanism of MAPKK-independent activation of p38 MAPK (Ge et al. 2002). TAB1 binds and activates TAK1, a MAP3K that can activate both the JNK and p38 MAPK pathways. However, TAB1 also binds p38 MAPK and causes MAPKK-independent activation by causing p38 MAPK autophosphorylation and activation. Evidence that this function of TAB1 is independent of TAK1 has been obtained from the identification of the splice variant TAB1 $\beta$ that does not bind TAK1, but does bind and activate p38 MAPK (Ge et al. 2003). The MAPKK-independent activation of $\mathrm{p} 38$ MAPK caused by TAB1 has been proposed to regulate the basal activity of p38 MAPK and to contribute to the activation of p38 MAPK by cell surface receptors, including TNF receptors $/ \mathrm{Ge}$ et al. 2002, 2003). The observation that TNF $\alpha$ does not activate p38 MAPK in $\mathrm{Mkk3}^{-/-} \mathrm{Mkk6}^{-/-}$fibroblasts suggests that TAB1 does not contribute to TNF $\alpha$-stimulated p38 MAPK activation in this cell type. Further studies are required to determine the physiological context of TAB1-mediated p38 MAPK activation. Importantly, the recent description of $\mathrm{Tab1}^{-/-}$mice, which die during early embryogenesis with cardiovascular and lung dysmorphogenesis, will facilitate this analysis (Komatsu et al. 2002).

\section{The MKK3/6 pathway regulates the cell cycle}

The p38 MAPK pathway has been reported to inhibit cell cycle progression by at least three different mechanisms (Bulavin et al. 2002a). First, p38 MAP kinase inhibits the expression of D-type cyclins (Lavoie et al. 1996). Second, p38 MAPK can phosphorylate and inhibit Cdc25B and Cdc25C, two protein phosphatases that activate cyclindependent protein kinase activity (Bulavin et al. 2001). Third, p38 MAP kinase phosphorylates the p53 tumor suppressor on two activating sites in the N-terminal re- 
Brancho et al.
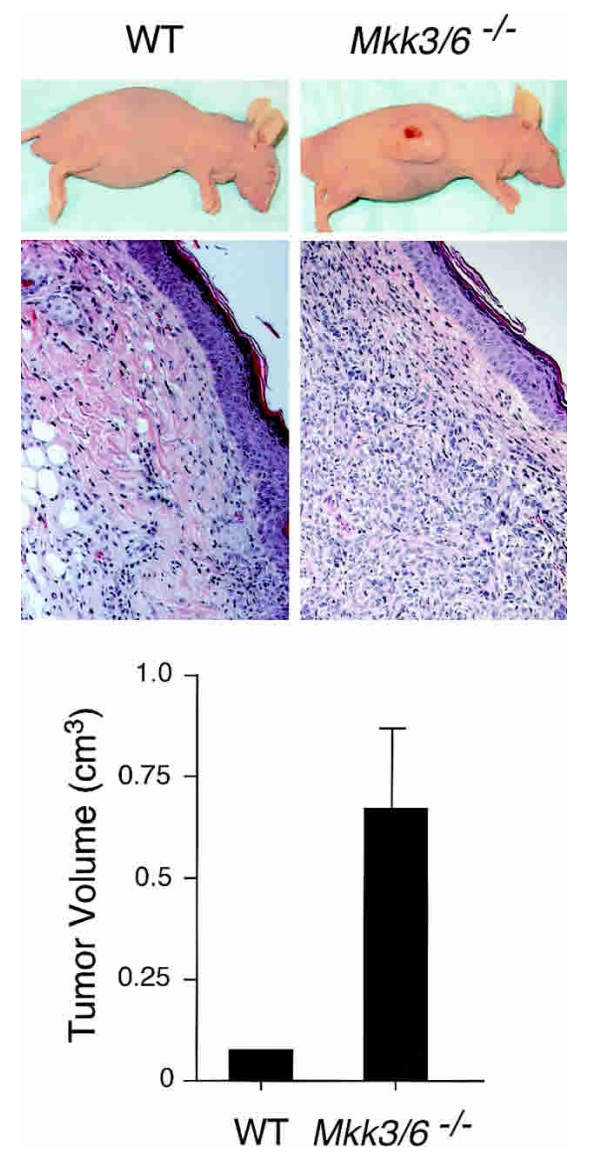

Figure 7. Compound disruption of $M k k 3$ and $M k k 6$ causes increased tumorigenesis. Wild-type (WT) and $\mathrm{Mkk3}^{-/-} \mathrm{Mkk6}^{-/-}$ fibroblasts immortalized with SV40 large T antigen were injected subcutaneously in athymic nude mice. Representative mice with tumors are illustrated. The mice were euthanized, and the tumors were fixed and processed for histological analysis. Sections of the tumors stained with H\&E are shown. The tumor volume was measured and is presented graphically (mean \pm S.D.; $n=5$ ).

gion (Ser 33 and Ser 46) and causes p53-dependent growth arrest (Bulavin et al. 1999; Sanchez-Prieto et al. 2000). Together, these targets of the p38 MAP kinase pathway (cyclin D, Cdc25, and p53) may cooperate to arrest the cell cycle. This finding suggests that defects in p38 MAPK function may contribute to cell cycle defects and increased tumorigenesis. Indeed, the Ppm1D gene (which encodes a phosphatase that inhibits p38 MAPK) is amplified in many human tumors (Bulavin et al. $2002 \mathrm{~b}$. Inactivation of p38 MAPK by gene targeting in mice or by overexpression of PPM1D dramatically increases tumorigenesis (Bulavin et al. 2002b). Similarly, in this study we found that decreased p38 MAPK activity caused by compound mutations of $M k k 3$ and $M k k 6$ causes growth arrest defects (Fig. 6) and increased tumorigenesis (Fig. 7).

\section{Conclusions}

The results of this study indicate that p38 MAPK is regulated by MKK3, MKK4, and MKK6. The MKK3 and
MKK6 protein kinases are specific activators of p38 MAPK. In contrast, MKK4 represents a site of integration of stress-activated MAPK pathways because it can activate both JNK and p38 MAPK. The repertoire of protein kinases that contribute to p38 MAPK activation depends on the specific stimulus that is examined. Thus, exposure of cells to TNF causes p38 MAPK activation by a mechanism that requires MKK3 and MKK6. In contrast, MKK3, MKK4, and MKK6 contribute to p38 MAPK activation caused by UV radiation. This role of MKK4 in p38 MAPK activation in fibroblasts is largely redundant with MKK3 and MKK6.

The contribution of MKK3, MKK4, and MKK6 to p38 MAPK activation may depend on the cell type that is examined. For example, differences in the expression of MKK3 and MKK6 account for the observation that Tcell-receptor-mediated p38 MAPK activation is selectively defective in $\mathrm{Mkk6}^{-/}$thymocytes and $\mathrm{Mkk3}^{-/-}$ $\mathrm{CD}^{+}$peripheral T-cells, respectively (Lu et al. 1999; Tanaka et al. 2002). Similarly, MKK4 may be an important activator of p38 MAPK in cells with low levels of MKK3 and MKK6.

The observation that p38 MAPK is activated by three different protein kinases suggests that this pathway represents a site of signal integration during the response of cells to cytokines, growth factors, and environmental stimuli. This is likely to be biologically significant because of the role of p38 MAPK in the regulation of cell cycle progression (Bulavin et al. 2002a). Indeed, defects in the p38 MAPK pathway are associated with tumorigenesis (Bulavin et al. 2002b).

\section{Materials and methods}

Mice

$\mathrm{Mkk3}^{-/-}$mice (Lu et al. 1999; Wysk et al. 1999), $\mathrm{Mkk}^{-/-}$mice (Yang et al. 1997), and $M k^{2} 6^{-1-}$ mice (Tanaka et al. 2002) have been described. $\mathrm{Mkk3}^{-1-} \mathrm{Mkk6}^{-1-}$ mice were obtained by interbreeding $\mathrm{Mkk3}^{-1-}$ and $\mathrm{Mkk}^{-1-}$ mice. Tumor assays were performed using 12-week-old male athymic nude mice (Charles River) by subcutaneous injection of $1 \times 10^{6}$ fibroblasts. All animals were housed in a facility accredited by the American Association for Laboratory Animal Care (AALAC), and the animal studies were approved by the Institutional Animal Care and Use Committee (IACUC) of the University of Massachusetts.

\section{Cell culture}

Murine embryo fibroblasts were isolated and cultured in Dulbecco's modified Eagle's medium supplemented with 10\% fetal calf serum (Invitrogen). The fibroblasts were immortalized using the SV40-large-T-antigen expression vector $\mathrm{p} 321-\mathrm{T}$ and the Fugene reagent. In vitro fibroblast proliferation assays were performed by staining with crystal violet (Tournier et al. 2001).

\section{siRNA studies}

Fibroblasts were transfected with double-stranded RNA (Dharmacon Research) targeting the sequence AATGCGGAGTAGT GATTGATTGCCCAT using Lipofectamine 2000 (Invitrogen), following the manufacturer's recommendations (Elbashir et al. 
2001). The double-stranded siRNA was designed to selectively suppress expression of mouse MKK4. Control experiments were performed using double-stranded RNA targeting the sequence AAACATGCAGAAAATGCTGTT, which suppresses the expression of luciferase (Elbashir et al. 2001). The cells were examined $48 \mathrm{~h}$ posttransfection.

\section{Biochemical assays}

Cells were lysed in Triton lysis buffer containing $20 \mathrm{mM}$ Tris (pH 7.4), $1 \%$ Triton X-100, $10 \%$ glycerol, $137 \mathrm{mM} \mathrm{NaCl}, 2 \mathrm{mM}$ EDTA, $25 \mathrm{mM} \beta$-glycerophosphate, $1 \mathrm{mM}$ sodium orthovanadate, $1 \mathrm{mM}$ phenymethylsulfonyl fluoride, and $10 \mu \mathrm{g} / \mathrm{mL}$ of aprotinin and leupeptin. Extracts (50 $\mu \mathrm{g}$ of protein) were examined by protein immunoblot analysis by probing with antibodies to JNK (PharMingen), phospho-JNK (Cell Signaling), p38 MAPK (Santa Cruz), phospho-p38 MAPK (Cell Signaling), ERK1/2 (Santa Cruz), phospho-ERK (Cell Signaling), MKK3 (Pharmingen), phospho-MKK3/6 (Cell Signaling), MKK4 (Santa Cruz), MKK6 (Stressgen), MKK7 (Pharmingen), Rb (Pharmingen), hypo-pRb (Pharmingen), and $\alpha$-tubulin (Sigma). Immunocomplexes were detected by enhanced chemiluminescence (NEN). MAP kinase activity was measured by in vitro kinase assays (Raingeaud et al. 1995). Ribonuclease protection assays were performed using reagents obtained from Pharmingen.

\section{Acknowledgments}

We thank Kathy Gemme for expert administrative assistance. This study was supported, in part, by a grant from the National Cancer Institute. R.J.D. and R.A.F. are investigators of the Howard Hughes Medical Institute.

The publication costs of this article were defrayed in part by payment of page charges. This article must therefore be hereby marked "advertisement" in accordance with 18 USC section 1734 solely to indicate this fact.

\section{References}

Adams, R.H., Porras, A., Alonso, G., Jones, M., Vintersten, K., Panelli, S., Valladares, A., Perez, L., Klein, R., and Nebreda, A.R. 2000. Essential role of p38 $\alpha$ MAP kinase in placental but not embryonic cardiovascular development. Mol. Cell 6: $109-116$.

Allen, M., Svensson, L., Roach, M., Hambor, J., McNeish, J., and Gabel, C.A. 2000. Deficiency of the stress kinase p38 $\alpha$ results in embryonic lethality: Characterization of the kinase dependence of stress responses of enzyme-deficient embryonic stem cells. J. Exp. Med. 191: 859-870.

Bardwell, L. and Thorner, J. 1996. A conserved motif at the amino termini of MEKs might mediate high-affinity interaction with the cognate MAPKs. Trends Biochem. Sci. 21: 373-374.

Bulavin, D.V., Saito, S., Hollander, M.C., Sakaguchi, K., Anderson, C.W., Appella, E., and Fornace Jr., A.J. 1999. Phosphorylation of human p53 by p38 kinase coordinates $\mathrm{N}$-terminal phosphorylation and apoptosis in response to UV radiation. EMBO J. 18: 6845-6854.

Bulavin, D.V., Higashimoto, Y., Popoff, I.J., Gaarde, W.A., Basrur, V., Potapova, O., Appella, E., and Fornace Jr., A.J. 2001. Initiation of a G2/M checkpoint after ultraviolet radiation requires p38 kinase. Nature 411: 102-107.

Bulavin, D.V., Amundson, S.A., and Fornace, A.J. 2002a. p38 and Chk1 kinases: Different conductors for the G2/M checkpoint symphony. Curr. Opin. Genet. Dev. 12: 92-97.

Bulavin, D.V., Demidov, O.N., Saito, S., Kauraniemi, P., Phillips, S., Amundson, S.A., Ambrosino, C., Sauter, G., Nebreda, A.R., Anderson, C.W., et al. 2002b. Amplification of PPM1D in human tumors abrogates p53 tumor-suppressor activity. Nat. Genet. 31: 210-215.

Chang, C.I., Xu, B.E., Akella, R., Cobb, M.H., and Goldsmith, E.J. 2002. Crystal structures of MAP kinase p38 complexed to the docking sites on its nuclear substrate MEF2A and activator MKK3b. Mol. Cell 9: 1241-1249.

Chiariello, M., Marinissen, M.J., and Gutkind, J.S. 2000. Multiple mitogen-activated protein kinase signaling pathways connect the cot oncoprotein to the c-jun promoter and to cellular transformation. Mol. Cell. Biol. 20: 1747-1758.

Derijard, B., Hibi, M., Wu, I.H., Barrett, T., Su, B., Deng, T., Karin, M., and Davis, R.J. 1994. JNK1: A protein kinase stimulated by UV light and Ha-Ras that binds and phosphorylates the c-Jun activation domain. Cell 76: 1025-1037.

Derijard, B., Raingeaud, J., Barrett, T., Wu, I.H., Han, J., Ulevitch, R.J., and Davis, R.J. 1995. Independent human MAPkinase signal transduction pathways defined by MEK and MKK isoforms. Science 267: 682-685.

Doza, Y.N., Cuenda, A., Thomas, G.M., Cohen, P., and Nebreda, A.R. 1995. Activation of the MAP kinase homologue RK requires the phosphorylation of Thr-180 and Tyr-182 and both residues are phosphorylated in chemically stressed $\mathrm{KB}$ cells. FEBS Lett. 364: 223-228.

Elbashir, S.M., Harborth, J., Lendeckel, W., Yalcin, A., Weber, K., and Tuschl, T. 2001. Duplexes of 21-nucleotide RNAs mediate RNA interference in cultured mammalian cells. $\mathrm{Na}$ ture 411: 494-498.

Enslen, H. and Davis, R.J. 2001. Regulation of MAP kinases by docking domains. Biol. Cell 93: 5-14.

Enslen, H., Brancho, D.M., and Davis, R.J. 2000. Molecular determinants that mediate selective activation of p38 MAP kinase isoforms. EMBO J. 19: 1301-1311.

Ganiatsas, S., Kwee, L., Fujiwara, Y., Perkins, A., Ikeda, T., Labow, M.A., and Zon, L.I. 1998. SEK1 deficiency reveals mitogen-activated protein kinase cascade crossregulation and leads to abnormal hepatogenesis. Proc. Natl. Acad. Sci. 95: 6881-6886.

Ge, B., Gram, H., Di Padova, F., Huang, B., New, L., Ulevitch, R.J., Luo, Y., and Han, J. 2002. MAPKK-independent activation of p38 $\alpha$ mediated by TAB1-dependent autophosphorylation of $\mathrm{p} 38 \alpha$. Science 295: 1291-1294.

Ge, B., Xiong, X., Jing, Q., Mosley, J.L., Filose, A., Bian, D., Huang, S., and Han, J. 2003. TAB1 $\beta$ (transforming growth factor- $\beta$-activated protein kinase 1 -binding protein $1 \beta$ ), a novel splicing variant of TAB1 that interacts with p38 $\alpha$ but not TAK1. J. Biol. Chem. 278: 2286-2293.

Giroux, S., Tremblay, M., Bernard, D., Cardin-Girard, J.F., Aubry, S., Larouche, L., Rousseau, S., Huot, J., Landry, J., Jeannotte, L., et al. 1999. Embryonic death of Mek1-deficient mice reveals a role for this kinase in angiogenesis in the labyrinthine region of the placenta. Curr. Biol. 9: 369-372.

Han, J., Jiang, Y., Li, Z., Kravchenko, V.V., and Ulevitch, R.J. 1997. Activation of the transcription factor MEF2C by the MAP kinase p38 in inflammation. Nature 386: 296-299.

Hazzalin, C.A., Cuenda, A., Cano, E., Cohen, P., and Mahadevan, L.C. 1997. Effects of the inhibition of p38/RK MAP kinase on induction of five fos and jun genes by diverse stimuli. Oncogene 15: 2321-2331.

Kishimoto, H., Nakagawa, K., Watanabe, T., Kitagawa, D., Momose, H., Seo, J., Nishitai, G., Shimizu, N., Ohata, S., Tanemura, S., et al. 2003. Different properties of SEK1 and MKK7 
in dual phosphorylation of stress-induced activated protein kinase SAPK/JNK in embryonic stem cells. J. Biol. Chem. 278: 16595-16601.

Komatsu, Y., Shibuya, H., Takeda, N., Ninomiya-Tsuji, J., Yasui, T., Miyado, K., Sekimoto, T., Ueno, N., Matsumoto, K., and Yamada, G. 2002. Targeted disruption of the Tabl gene causes embryonic lethality and defects in cardiovascular and lung morphogenesis. Mech. Dev. 119: 239-249.

Kyriakis, J.M. and Avruch, J. 2001. Mammalian mitogen-activated protein kinase signal transduction pathways activated by stress and inflammation. Physiol. Rev. 81: 807-869.

Lavoie, J.N., L'Allemain, G., Brunet, A., Muller, R., and Pouyssegur, J. 1996. Cyclin D1 expression is regulated positively by the p42/p44MAPK and negatively by the p38/HOGMAPK pathway. J. Biol. Chem. 271: 20608-2016.

Lawler, S., Fleming, Y., Goedert, M., and Cohen, P. 1998. Synergistic activation of SAPK1/JNK1 by two MAP kinase kinases in vitro. Curr. Biol. 8: 1387-1390.

Lin, A., Minden, A., Martinetto, H., Claret, F.X., Lange-Carter, C., Mercurio, F., Johnson, G.L., and Karin, M. 1995. Identification of a dual specificity kinase that activates the Jun kinases and p38-Mpk2. Science 268: 286-290.

Lu, H.T., Yang, D.D., Wysk, M., Gatti, E., Mellman, I., Davis, R.J., and Flavell, R.A. 1999. Defective IL-12 production in mitogen-activated protein (MAP) kinase kinase 3 (Mkk3)deficient mice. $E M B O$ J. 18: 1845-1857.

Nishina, H., Fischer, K.D., Radvanyi, L., Shahinian, A., Hakem, R., Rubie, E.A., Bernstein, A., Mak, T.W., Woodgett, J.R., and Penninger, J.M. 1997. Stress-signalling kinase Sek1 protects thymocytes from apoptosis mediated by CD95 and CD3. Nature 385: 350-353.

Raingeaud, J., Gupta, S., Rogers, J.S., Dickens, M., Han, J., Ulevitch, R.J., and Davis, R.J. 1995. Pro-inflammatory cytokines and environmental stress cause p38 mitogen-activated protein kinase activation by dual phosphorylation on tyrosine and threonine. J. Biol. Chem. 270: 7420-7426.

Raingeaud, J., Whitmarsh, A.J., Barrett, T., Derijard, B., and Davis, R.J. 1996. MKK3- and MKK6-regulated gene expression is mediated by the p38 mitogen-activated protein kinase signal transduction pathway. Mol. Cell. Biol. 16: 1247-1255.

Sanchez-Prieto, R., Rojas, J.M., Taya, Y., and Gutkind, J.S. 2000. A role for the p38 mitogen-activated protein kinase pathway in the transcriptional activation of p53 on genotoxic stress by chemotherapeutic agents. Cancer Res. 60: 2464-2472.

Schaeffer, H.J. and Weber, M.J. 1999. Mitogen-activated protein kinases: Specific messages from ubiquitous messengers. Mol. Cell. Biol. 19: 2435-2444.

Tamura, K., Sudo, T., Senftleben, U., Dadak, A.M., Johnson, R., and Karin, M. 2000. Requirement for p38 $\alpha$ in erythropoietin expression: A role for stress kinases in erythropoiesis. Cell 102: 221-231.

Tanaka, N., Kamanaka, M., Enslen, H., Dong, C., Wysk, M., Davis, R.J., and Flavell, R.A. 2002. Differential involvement of p38 mitogen-activated protein kinase kinases MKK3 and MKK6 in T-cell apoptosis. EMBO Rep. 3: 785-791.

Tournier, C., Whitmarsh, A.J., Cavanagh, J., Barrett, T., and Davis, R.J. 1999. The MKK7 gene encodes a group of c-Jun $\mathrm{NH}_{2}$-terminal kinase kinases. Mol. Cell. Biol. 19: 15691581.

Tournier, C., Dong, C., Turner, T.K., Jones, S.N., Flavell, R.A., and Davis, R.J. 2001. MKK7 is an essential component of the JNK signal transduction pathway activated by proinflammatory cytokines. Genes \& Dev. 15: 1419-1426.

Wada, T., Nakagawa, K., Watanabe, T., Nishitai, G., Seo, J., Kishimoto, H., Kitagawa, D., Sasaki, T., Penninger, J.M.,
Nishina, H., et al. 2001. Impaired synergistic activation of stress-activated protein kinase SAPK/JNK in mouse embryonic stem cells lacking SEK1/MKK4: Different contribution of SEK2/MKK7 isoforms to the synergistic activation. J. Biol. Chem. 276: 30892-30897.

Weston, C.R., Lambright, D.G., and Davis, R.J. 2002. Signal transduction. MAP kinase signaling specificity. Science 296: 2345-2347.

Wysk, M., Yang, D.D., Lu, H.T., Flavell, R.A., and Davis, R.J. 1999. Requirement of mitogen-activated protein kinase kinase 3 (MKK3) for tumor necrosis factor-induced cytokine expression. Proc. Nat1. Acad. Sci. 96: 3763-3768.

Yang, D., Tournier, C., Wysk, M., Lu, H.T., Xu, J., Davis, R.J., and Flavell, R.A. 1997. Targeted disruption of the MKK4 gene causes embryonic death, inhibition of c-Jun $\mathrm{NH}_{2}$-terminal kinase activation, and defects in AP-1 transcriptional activity. Proc. Natl. Acad. Sci. 94: 3004-3009. 


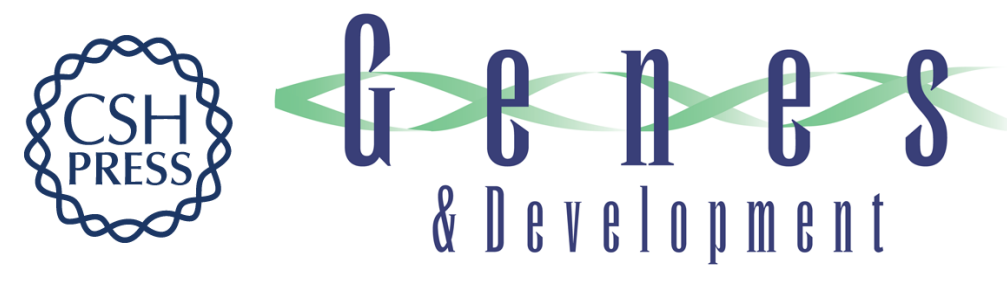

\section{Mechanism of p38 MAP kinase activation in vivo}

Deborah Brancho, Nobuyuki Tanaka, Anja Jaeschke, et al.

Genes Dev. 2003, 17:

Access the most recent version at doi:10.1101/gad.1107303

References This article cites 41 articles, 22 of which can be accessed free at: http://genesdev.cshlp.org/content/17/16/1969.full.html\#ref-list-1

License

Email Alerting Receive free email alerts when new articles cite this article - sign up in the box at the top Service right corner of the article or click here.

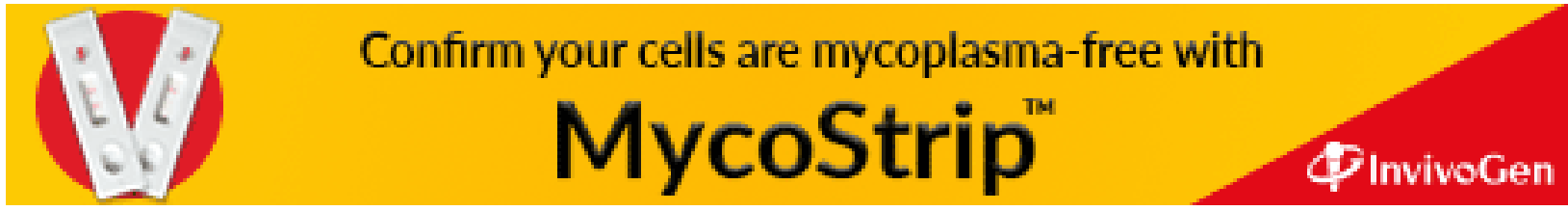

